\title{
Interfaces digitais de serviços de entrega de alimentos: análise semiótica dos aplicativos da plataforma iFood
}

\author{
Digital interfaces of food delivery services: \\ semiotics analysis of iFood platform applications
}

cultura material, semiótica, plataformas digitais, design da informação

\section{Ana Carolina Ribeiro Ferreira da Costa, Leandro Manuel Reis Velloso}

material culture, semiotic, digital platforms, information design

\begin{abstract}
O artigo investiga a relação entre interfaces de plataformas digitais, e os diferentes agentes que conecta, através de análises semióticas seguindo bases peirceanas. Como estudo de caso, foram analisados os aplicativos do Cliente Final e do Entregador da plataforma de entregas de comida iFood, notável por sua grande base de usuários ativos em território brasileiro. Após a seleção de pontos chave de interação entre plataforma e usuário, foram minuciosamente descritos os elementos encontrados em cada tela seguindo um roteiro de análises que auxiliou o exame do signo segundo sua natureza triádica proposta por Peirce. Com os resultados obtidos foi possível concluir que a experiência e as interações proporcionadas pelos aplicativos sugerem que cada um dos agentes ocupe um papel social específico e predeterminado pela plataforma.
\end{abstract}

Through a Peircean-based semiological analysis, this article investigates the relations between digital platform interfaces and different social agents. This case study was the Client and Deliveryperson versions apps of the food delivery platform iFood, notable for its large base of active users in Brazilian territory. After selecting key points of interaction between platform and users, the elements on screen was thoroughly described - guided by an analysis script that helped reveal the triadic nature of a sign, as proposed by Peirce. The results enabled to conclude that the experience and interactions within the application prompt each social agent to occupy a specific, predetermined social role.

\section{Introdução}

Os objetos criados condicionam a própria existência humana compondo um cenário que conscientiza aquilo que é apropriado e o que não é e, em última instância, determinam a possibilidade de realizar ações. Por rodear indivíduos ao longo de toda sua vida, os objetos têm papel fundamental no processo de formação social 
regendo os comportamentos das pessoas através dos cenários que produzem(MILLER, 2013).

A partir do momento no qual a existência de um artefato é compreendida como pressuposta, ou seja, torna-se inimaginável uma vida sem tal artefato, a existência humana é condicionada a eles de forma a alienar-se aos objetos. Passa a não haver mais uma racionalização sobre a existência deles, nem do porquê nem de como foram criados. Quanto menos a presença de um objeto é percebida, mais intenso será o processo de alienação (MILLER, 2013).

Através da interação com artefatos são formadas as interfaces entre os seres humanos e o mundo. Tal ideia pode ser melhor entendida através do diagrama ontológico do design proposto por Bonsiepe (1997, p. 10), que determina a existência de três domínios conectados por uma categoria central: o primeiro diz respeito ao usuário, agente social que deseja cumprir um objetivo; o segundo é o próprio objetivo ou tarefa que o usuário deseja cumprir; e, em terceiro lugar, a ferramenta ou informação, que intermedeia a concretude da ação. Essas três instâncias são articuladas através de uma interface, que é a instância central proposta pelo diagrama. É de se imaginar, portanto, que o resultado de realizar a tarefa pretendida se dará a partir do desempenho conjunto entre usuário e artefato. Durante o processo de criação o designer exerce sua influência sobre o artefato manipulando suas propriedades, podendo provocar alterações, conscientes ou não, no comportamento dos usuários (FOKKINGA, 2020).

Tratando-se do campo do design da informação, o processo de criação de interfaces que medeiam a informação percebida pelo usuário têm primordial importância para a aquisição de conhecimento e contribui para a possibilidade de ação dos indivíduos. É parte do escopo de trabalho do campo organizar os dados receptíveis e perceptíveis de forma a colaborar com a transformação de uma estrutura em um estado altamente entrópico, desordenado, para um novo estado de baixa entropia, ordenado, de forma a criar condições para que o usuário seja capaz de compreender os dados apresentados e utilizá-los (BONSIEPE, 2011).

Dessa maneira, a transformação realizada pelo trabalho do designer não só é fundamental para garantir que os usuários possam realizar as tarefas que desejam, mas também permite que sejam desempenhadas ações mais eficientes, indicando, dessa forma, como o usuário as executará. O paradigma do design centrado no humano se baseia em técnicas que buscam compreender as necessidades, desejos e experiências das pessoas com os produtos, sistemas e serviços, tornando-os mais intuitivos em seus aspectos físicos, cognitivos e emocionais (GIACOMIN, 2014). Porém, como viu-se anteriormente, a utilização dos artefatos está associada a um processo de alienação, onde as pessoas não só fazem o que desejam com os objetos, mas também aquilo que os objetos sugerem. No design para a web essa modulação da experiência é evidente, pois, em vista de satisfazer objetivos que vão além das necessidades do usuário, o designer projeta 
jornadas de uso ideais que combatem os pontos de desconforto (as dores) que o usuário eventualmente poderá sentir ao longo do processo, fazendo com que o uso do artefato seja sempre positivo (KALBACH, 2017; KRUG, 2006).

A websérie Dopamine (FAVIER, 2019) demonstra como o funcionamento do aplicativo Uber é pensado de modo a estimular a produção do hormônio dopamina, responsável pela sensação de prazer e motivação, associados à dependência. O documentário mostra como as dinâmicas de uso do aplicativo reduz o poder de tomada de decisão pelo usuário, restringindo seu tempo de resposta às chamadas. Um exemplo inverso é dado por Miller (2013, p. 174) sobre o uso de redes sociais de forma a agilizar o debate entre comunidades religiosas dispersas de Trinidad, garantindo o tempo de reflexão ideal aos usuários.

\subsection{Plataformas digitais e seus aplicativos}

Antes de prosseguir, é importante compreender como os aplicativos fazem parte de um sistema maior e mais complexo que são as plataformas digitais. Não há um consenso sobre a definição exata do que são essas plataformas, é possível encontrar na literatura diversas maneiras de conceituá-las. No entanto, há uma característica em comum descrita por vários autores: plataformas promovem e intermedeiam a relação entre diferentes atores sociais (VALENTE, 2020; EVANS; SCHMALENSEE, 2016 apud GRABHER, 2020; MARK et al., 2018). Esse parece ser o princípio fundante de uma plataforma, o que também se comprova em declarações publicitárias e em termos de uso de grandes plataformas digitais: "Nossa tecnologia [...] já possibilitou bilhões de conexões entre pessoas no mundo todo" (UBER, 2020); “[...] mantemos um sistema de mensagens inteligente para que anfitriões e hóspedes possam se comunicar com segurança” (AIRBNB, 2020); "O serviço do ifood.com.br consiste, portanto, em aproximar, através do nosso site, os USUÁRIOS e os RESTAURANTES cadastrados, possibilitando que os USUÁRIOS encaminhem, aOS RESTAURANTES, pedidos de entrega de gêneros alimentícios" (IFOOD, 2020).

É por conta de tal característica que plataformas se beneficiam do efeito de rede: quanto mais usuários fornecedores estão conectados, mais usuários consumidores estão interessados em utilizar a mesma plataforma e vice-versa. É nessa lógica de que o ganhador leva tudo que as plataformas se tornam ainda mais interessantes aos usuários e

1 Em 2014 o preço mínimo da tarifa do Uber chegou a us $\$ 100,00$ durante episódio de sequestro em um bairro comercial de Sydney, que havia culminado em 3 mortes e gerado pânico na população. aumentam seu valor de mercado (PARKER et al., 2016 apud GRABHER, 2020). Apesar de se declararem apenas como intermediadores e facilitadores do processo, foi possível notar que tal intermediação não é neutra, as plataformas empregam diversas práticas comerciais favorecendo o seu próprio negócio (GRABHER, 2020, p. 4; VALENTE, 2020, p. 81), como exemplifica Tom Slee (2017, p. 265) sobre os "preços dinâmicos" cobrados pela Uber em momentos de crise. ${ }^{1}$ 
A ação da plataforma se dá por meio de infraestruturas digitais que são tangibilizadas através de "serviços e aplicações que se espalham por toda a atividade social na forma de aplicativos, automatizando decisões e modulando comportamentos" (VAlente, 2020, p. 82). Além da importância dessas infraestruturas, adiciona-se o fato de que "já se afirmou que, do ponto de vista do usuário, a interface é sinônimo de programa” (BONSIEPE, 1997, p. 47), ou seja, o usuário encara aquilo que vê e interage como sendo o total do sistema e, dessa forma, é possível o paralelo demonstrando como o aplicativo é percebido como sinônimo da plataforma.

\subsection{O contexto das plataformas de delivery}

A crise sanitária iniciada em 2020 demonstrou um cenário fértil para empresas de entrega, como mostram os dados apresentados na conferência Brazil at Silicon Valley pelo Google for Startups (2020): em comparação com dados do ano anterior, a procura por serviços de deliveries subiu $72 \%$ entre os meses de março e abril e algumas empresas de entrega tiveram crescimento exponencial no mesmo período. Contudo, observou-se que a utilização de serviços digitais de entrega de comida já estava em franco crescimento mesmo antes do período de crise sanitária, como aponta o relatório realizado pelo Instituto Foodservice Brasil (2019).

Se por um lado a demanda de pedidos via aplicativos aumenta, de outro, aumenta também o número de pessoas que passam a trabalhar como entregadores de aplicativos. Em 2018 houve um crescimento de $29,2 \%$ da população de trabalhadores em veículos, incluindo motoristas de aplicativo, taxistas, motoristas e trocadores de ônibus, o que significa um acréscimo de 810 mil pessoas na atividade, totalizando 3,9 milhões de pessoas (PNAD, 2019).

\section{0 caso iFood}

Esse artigo trata sobre o caso do aplicativo iFood, uma plataforma de delivery de grande relevância para o contexto brasileiro. $\mathrm{O}$ aplicativo foi o segundo mais acessado na categoria de Comidas e Bebidas durante o ano de 2019 entre os brasileiros (SIMILARWEB, 2020), perdendo apenas para a plataforma Tudo Gostoso que não oferece os mesmos serviços e, portanto, não faz concorrência direta. O iFood, que desde 2013 pertence ao grupo empresarial Movile, é uma empresa brasileira fundada no ano de 2011. Em 2016 expandiu suas operações para outros países da América Latina e declara estar presente no México, Colômbia e Argentina com uma base de 5,1 milhões de usuários ativos, gerenciando 30,6 milhões de pedidos mensais (MOVILE, 2020).

Apesar de atuar em vários segmentos da cadeia de alimentação fora de casa, seu modelo principal de negócio prevê a articulação de 
três grupos principais de usuários intermediados via infraestrutura digital: 1) os clientes finais que solicitam a entrega, nomeados como Clientes; 2) os restaurantes que fornecem as refeições, chamados por Restaurantes; e 3) os entregadores que levam a encomenda produzida pelos Restaurantes até o local indicado pelos Clientes, nomeados como Entregadores. Para cada grupo, a plataforma iFood tem um modelo de relacionamento diferente, demonstrado em três contratos de uso distintos. Ao investigar o relacionamento entre plataforma e dois de seus grupos de usuários, Clientes e Entregadores, buscou-se também compreender como a própria empresa declara se relacionar com esses agentes. As descrições dos modelos de relacionamento apresentadas a seguir estão disponibilizadas pela própria companhia em seus websites.

Para os Clientes, a plataforma declara-se como intermediadora do processo de compra oferecendo opção de pagamento e isentando-se de quaisquer responsabilidades com a qualidade do alimento, preparo ou entrega do pedido. Entre as responsabilidades do iFood estão: fornecer o ambiente virtual que possibilita o cadastro, compra e pagamento de pedidos de alimentos; e garantir a segurança dos dados armazenados de seus usuários (IFOOD, 2020a).

Já para os Entregadores a plataforma esclarece mais uma vez seu papel de intermediadora e os considera profissionais independentes que atuam "como parte da operacionalização da atividade de agenciamento e intermediação do iFood” (IFOOD, 2020b). Declaram ainda que os valores pagos aos Entregadores não têm relação com os valores de taxa de serviços ou de entrega pagos pelos Clientes e que, por não ser uma empresa de logística, seu compromisso se limita ao fornecimento da plataforma tecnológica. Aos Entregadores, cabe o reconhecimento de que são independentes e não exclusivos e, portanto, não firmam qualquer vínculo subordinativo com o iFood (IFOOD, 2020b).

Através de uma análise semiótica peirceana, foram analisados os aplicativos dos usuários Clientes e dos Entregadores observando de que maneira o cenário proposto acima é tangibilizado através das interfaces digitais. Foi possível, então, compreender como se dá a interação entre aplicativo e usuário, entre Clientes e Entregadores, e como o aplicativo influencia as ações de seus usuários.

\section{Metodologia da análise semiótica}

O entendimento sobre a percepção semiótica, assim como estudos de questões culturais, já foi apontado, e tem sido trabalhado, como um importante foco na pesquisa voltada para avaliação de usabilidade em aplicativos e outros aspectos das interfaces digitais (ISLAM, 2013).

Baseando-se no proposto de que as formas dos artefatos não possuem um significado fixo, mas são expressas em um processo de significação (CARDOSO, 2016), observou-se as possíveis interpretações que as telas dos aplicativos geram em seus usuários. Tendo como referência um estudo que analisou 186 casos de design organizando de 
modo triádico a relação entre usuário e artefato (FOKKINGA; DESMET; HEKKERT, 2020) e a natureza triádica do signo proposta por Peirce, conforme descrito por Santaella (2015), a análise semiótica sistemática das telas foi realizada em três instâncias:

- A primeira, tratando sobre o interno do próprio signo, foi analisada do ponto de vista qualitativo-icônico e convencionalsimbólico, sendo descritas as formas, cores e movimentos dos elementos ali dispostos;

- Para a segunda, compreendendo aquilo que o signo indica, representa ou se refere, foram descritos os objetos diretos e os dinâmicos de cada tela;

- Já na última instância, referindo-se aos tipos de efeitos interpretativos que o signo pode despertar nos usuários, foram descritos três níveis de interpretantes: o emocional, o energético e o lógico, seguindo a teoria peirceana.

Para a análise, foi utilizado um roteiro baseado em uma das estruturas propostas por Santaella (2015, p. 59-67) e, em cada etapa da análise, foram utilizadas perguntas guias que têm como referência a clínica de design apresentada por Bonsiepe (2011) para o estudo de interfaces digitais. O roteiro de análise semiótica segue conforme o Quadro 1.

Quadro 1 Roteiro para análises semióticas de interfaces digitais.

(Continua)

\begin{tabular}{|c|c|c|c|}
\hline \multirow[t]{6}{*}{$\begin{array}{l}\text { Primeira instância } \\
\text { de análise }\end{array}$} & \multirow[t]{5}{*}{$\begin{array}{l}\text { Ponto de vista } \\
\text { qualitativo-icônico }\end{array}$} & Cores e imagens & $\begin{array}{l}\text { 1. Como se usam as cores? } \\
\text { 2. Elas são relacionadas com funcionalidades? Como? } \\
\text { 3. Como são semanticamente usadas? } \\
\text { 4. Têm boa visibilidade? } \\
\text { 5. Quais paletas cromáticas são usadas? } \\
\text { 6. Como é o tratamento de imagens (tratamento digital, } \\
\text { paletas cromáticas)? }\end{array}$ \\
\hline & & Formas & $\begin{array}{l}\text { 7. Qual a variedade de tipografia (tipo } \\
\text { de fonte, tamanhos, estilos)? } \\
\text { 8. Como se usam os elementos da identidade corporativa? } \\
\text { 9. Quais as formas dos botões e elementos padrão? }\end{array}$ \\
\hline & & $\begin{array}{l}\text { Elementos } \\
\text { no espaço }\end{array}$ & $\begin{array}{l}\text { 10. A Interface está dividida em zonas funcionais? Quais? } \\
\text { 11. Como são diferenciadas, hierarquizadas e enfatizadas } \\
\text { as informações? } \\
\text { 12. Como o layout está estruturado? }\end{array}$ \\
\hline & & $\begin{array}{l}\text { Gestos e } \\
\text { animações }\end{array}$ & $\begin{array}{l}\text { 13. Como se usam as animações? } \\
\text { 14. Qual a dosagem dos efeitos? } \\
\text { 15. Quais modos de interação o aplicativo oferece? }\end{array}$ \\
\hline & & Sons & 16. Como se usa o som? \\
\hline & \multicolumn{2}{|c|}{$\begin{array}{l}\text { Ponto de vista } \\
\text { convencional-simbólico }\end{array}$} & $\begin{array}{l}\text { 17. Qual é a forma da curva de aprendizagem? É coerente } \\
\text { com o grau de dificuldade crescente? } \\
\text { 18. A interface corresponde ao estado da arte? } \\
\text { 19. A interface é agradável, lúdica, aborrecida, complexa? } \\
\text { 20. A interface é coerente com seus objetivos? }\end{array}$ \\
\hline
\end{tabular}


Quadro 1 Roteiro para análises semióticas de interfaces digitais.

\begin{tabular}{lll}
\hline $\begin{array}{ll}\text { Segunda Instância } \\
\text { de análise }\end{array}$ & Objeto Imediato & 21. Qual o objeto imediato? \\
\cline { 2 - 3 } & Objeto dinâmico & 22. Qual(is) o(s) objeto(s) dinâmicos? \\
\hline $\begin{array}{l}\text { Terceira instância } \\
\text { de análise }\end{array}$ & Interpretante Emocional & $\begin{array}{l}\text { 23. Quais as emoções evocadas pela interface? } \\
\text { 24. A interface é capaz de produzir sentimentos? }\end{array}$ \\
\cline { 2 - 3 } & Interpretante Energético & $\begin{array}{l}\text { 25. Quais ações internas a interface suscita? } \\
\text { 26. Quais ações externas a interface suscita? }\end{array}$ \\
\cline { 2 - 3 } & Interpretante Lógico & 27. Quais as regras que precisam ser compreendidas para o \\
& & bom uso da interface? \\
\hline
\end{tabular}

\section{Resultados}

O material utilizado na análise foi composto por telas representativas de pontos considerados chave dentro da jornada de uso de ambos os usuários. Para os entregadores foram analisadas as telas: Oferecimento de trabalho, Percurso de entrega, Chat, Histórico de entregas e Remuneração. Para os Clientes foram analisadas as telas: Tela inicial, Chat, Tela de gorjeta, Tela de Avaliação.

O material utilizado foi gentilmente cedido pelas próprias equipes de design do iFood. Ao realizar algumas entrevistas pontuais não estruturadas com usuários para sanar dúvidas, foram encontradas diferenças entre a tela de chat do entregador enviada pela empresa e aquela que estava efetivamente disponível ao usuário em seu celular. A segunda foi escolhida para análise.

Como produto, obteve-se um quadro no qual foram cruzadas as perguntas do roteiro com as telas selecionadas. Dada a complexidade ainda presente nos dados obtidos, optou-se por destacar as informações de maior relevância e que pudessem constituir conhecimentos novos sobre os artefatos conforme descritos no Quadro 2.

\section{Discussão}

As análises se estruturaram a partir da observação e descrição dos elementos fundamentais da composição, como as cores e as formas encontradas. Em praticamente todas as telas as cores utilizadas eram neutras, estando o branco, o preto e o cinza muito presentes. Enquanto o branco era mais utilizado no fundo de telas, o preto e o cinza compunham as cores dos textos, dessa maneira as telas se revelavam bastante claras. Outras cores eram utilizadas para situações como demarcar elementos em seus diferentes estados, chamar atenção para determinadas informações e para indicar possibilidade de ações. Tais observações valem para ambos os aplicativos analisados. 
Quadro 2 Destaques dos resultados obtidos através das análises semióticas

\begin{tabular}{ll}
\hline Destaques & Descrições \\
\hline $\begin{array}{l}\text { Neutralidade das } \\
\text { cores da tela }\end{array}$ & $\begin{array}{l}\text { Predomina a cor branca como fundo, e as cores cinza e preto são } \\
\text { utilizadas para textos, produzindo telas bastante claras. }\end{array}$ \\
\hline $\begin{array}{l}\text { Uso da cor vermelha } \\
\text { da marca }\end{array}$ & $\begin{array}{l}\text { O tom vermelho típico da marca é usado para indicar ações nos } \\
\text { aplicativos e nunca associado a símbolos negativos. }\end{array}$ \\
\hline $\begin{array}{l}\text { Signos visuais e } \\
\text { sonoros da marca }\end{array}$ & $\begin{array}{l}\text { O logo da marca é pouco usado, porém, para entregadores, o aviso } \\
\text { sonoro de um novo pedido é a repetição musical da palavra ifood. }\end{array}$ \\
\hline $\begin{array}{l}\text { Direcionamentos na } \\
\text { tomada de decisão }\end{array}$ & $\begin{array}{l}\text { A interface evidencia alguns itens em detrimento de outros guiando } \\
\text { a tomada de decisão do usuário. }\end{array}$ \\
\hline $\begin{array}{l}\text { Ranqueamento de } \\
\text { restaurantes }\end{array}$ & $\begin{array}{l}\text { Dentro da lógica da plataforma são criados diversos modelos de } \\
\text { ranqueamento para os Restaurantes que aparecem ao Cliente. }\end{array}$ \\
\hline $\begin{array}{l}\text { Possibilidades } \\
\text { distintas de ações }\end{array}$ & $\begin{array}{l}\text { Enquanto Clientes têm controle sobre suas ações, os Entregadores } \\
\text { possuem menor capacidade de interferência no processo. }\end{array}$ \\
\hline $\begin{array}{l}\text { Modos de avaliação } \\
\text { Cada agente é avaliado por métodos distintos, exceto os Clientes. }\end{array}$ \\
\hline $\begin{array}{l}\text { Denúncia e gorjeta } \\
\text { As funcionalidades de Denúncia e Gorjeta evidenciam a posição e o } \\
\text { papel que se espera de cada um dos agentes da plataforma. }\end{array}$ \\
\hline
\end{tabular}

Quase não foram encontrados elementos que remetem à marca, em todas as telas analisadas estavam presentes apenas três itens mais relevantes:

1. O primeiro deles, e também mais explícito, é o som emitido para os Entregadores quando chega uma oferta de trabalho dizendo iFood. É interessante notar que tal sonido anuncia um momento muito esperado pelo usuário e que deve ser respondido em poucos segundos, caso contrário é perdida a chance de realizar aquela entrega. Assim, o som pode criar uma atmosfera de alerta e urgência para o Entregador.

2. O segundo aspecto que indica a empresa são algumas ilustrações do tipo emoji encontradas na tela de gorjeta visualizada pelo Cliente que são personalizados segundo a paleta cromática utilizada pela marca. Associam-se aos valores possíveis de doações e representam feições positivas.

3. O terceiro elemento de marca encontrado é a própria cor vermelha. Ela está normalmente associada a itens que possibilitam ações e nunca é relacionada com aspectos negativos como, por exemplo, falhas no pagamento. O caso é interessante pois em outras situações fora do contexto iFood espera-se que a cor vermelha indique algo problemático.

Analisando os elemento da tela em maior profundidade, entendeu-se como em cada tela é proposta a interatividade e uma diferença crucial foi notada entre os dois aplicativos analisados: 
enquanto nas telas dos Entregadores os componentes são muito mais informativos e sugerem menos interações, quase todos os elementos das telas dos Clientes são interativos, o que indica uma gama maior de ações possíveis para esse grupo. Para os Entregadores a ação mais importante dentro do aplicativo é a de aceitar ou rejeitar uma oferta de trabalho, enquanto o Cliente decide sobre o restaurante que fará o pedido, a forma de pagamento e gorjeta, podendo ainda avaliar os serviços prestados pelos outros agentes.

Ainda que a limitação de ações seja mais evidente para os Entregadores, todas as ações que ocorrem dentro da plataforma são mediadas de alguma maneira. Na tela inicial do Cliente notamos um direcionamento das ações de forma a influenciar a tomada de decisão. Essa tela tem como objetivo apresentar todas as opções de restaurantes e comidas disponíveis na plataforma para que o Cliente decida sobre o que irá pedir, por isso a tela apresenta uma espécie de vitrine dos vários restaurantes cadastrados. Existem muitas opções e algumas recebem maior destaque como, por exemplo, os restaurantes que têm compromisso de fidelidade com o iFood.

A tela inicial ainda exibe informações que, por estarem acessíveis, também podem ser ponderadas durante o processo de decisão, são elas:

- A classificação de 1 a 5 estrelas que o restaurante recebe dentro da plataforma, e que é feita por outros Clientes que já compraram daquele estabelecimento;

- Os selos de "Super Restaurante", dados a estabelecimentos que cumprem requisitos do iFood;

- A distância do restaurante até o endereço informado pelo Cliente;

- O tempo aproximado de entrega;

- Possibilidade de rastreamento em tempo real através da "Entrega iFood".

A influência exercida sobre o Cliente em sua tomada de decisão também é emblemática durante a etapa de avaliação, na qual o usuário é convidado a opinar sobre os serviços recebidos. Nela os métodos escolhidos para avaliação de cada prestador são diferentes: os restaurantes são avaliados de 1 a 5 , somente por sua comida; o Entregador recebe uma avaliação binária de "Sim, gostei [da entrega]" e "Não, [a entrega] poderia melhorar"; e o iFood é avaliado em uma escala de o a 10. O motivo da diferença de métodos não é declarado em nenhum momento e fica evidente que o Entregador recebe uma avaliação superficial se comparada às outras duas. Ainda nesta etapa, o usuário não é capaz de enviar mensagens livres e de sua autoria para o Entregador ou para o iFood, recurso disponível apenas na avaliação dos Restaurantes e se limita a 300 caracteres. As informações que os clientes são capazes de enviar aos outros usuários estão, na maioria dos casos, limitadas pelos critérios e formatos pré-estabelecidos pelo aplicativo. 
Se para o Cliente o aplicativo sugere ou induz decisões e ações, para o Entregador elas muitas vezes são ditadas. A título de exemplo:

- A oferta de trabalho aparece no dispositivo do Entregador interrompendo qualquer outra atividade que estiver sendo desempenhada no celular;

- Para decidir sobre a oferta de trabalho o usuário dispõe de poucos segundos, caso contrário a oferta é automaticamente cancelada;

- O caminho a ser percorrido entre os locais de coleta e de entrega final são apontados na tela do próprio aplicativo. Aqui o Entregador pode se utilizar de outros aplicativos parceiros para concluir a ação, no entanto é importante levantar que o aplicativo iFood Entregador está disponível apenas para dispositivos com sistema operacional do tipo Android e as versões mais baratas dos aparelhos dispõem de pouco espaço em memória, o que dificulta a instalação de muitos aplicativos;

- O Entregador só é capaz de finalizar a entrega no aplicativo quando está próximo do endereço indicado pelo Cliente.

Apesar de todo o esforço de direcionamento das ações dos usuários no aplicativo, há um ponto onde os usuários podem trocar informações livremente, essa interação é feita através da tela de chat. Os elementos estéticos e funcionais do chat em ambos os aplicativos são muito similares: as telas são claras e identificam os emissores das mensagens através de balões de fala com cores diferentes. O tempo de acesso a essa tela é restrito para ambos, estando disponível somente durante o período de entrega. Apesar de muito semelhantes, observa-se, no entanto, que na tela do Cliente existe um botão "denúncia" e o mesmo não aparece para o Entregador. Desta forma, tal elemento sugere que o primeiro está em um papel de indivíduo que precisa ser protegido do segundo, que, por sua vez, pode apresentar algum tipo de ameaça. É digno de nota compreender como o aplicativo, dessa maneira, desenha as relações entre os dois grupos de usuários.

Além do sugerido pelo botão "denúncia", há outras duas situações em que notou-se de forma veemente essas sugestões de papéis. $\mathrm{Na}$ primeira o Cliente é o único que tem o poder de avaliar a atuação dos outros usuários, ainda que nas condições já citadas acima, sem que seja avaliado. Dessa maneira ele é colocado em uma posição privilegiada em relação aos outros. A segunda situação que vale destaque, diz respeito à tela de gorjeta. Neste momento, todos os elementos apresentados tentam convencer o Cliente a doar um valor a mais para o Entregador e um dos argumentos apresentados é o de que o dinheiro doado, que pode ser no máximo dez reais, fará muita diferença para quem recebe, ou seja, o Cliente é capaz de fazer diferença para o Entregador. É interessante perceber como mais uma vez o Cliente tem um papel ativo frente ao Entregador, visto que o aplicativo intercede em nome deste. 


\section{Conclusão}

Como descreveu-se no início, conforme teorizado por Daniel Miller (2015), os objetos também têm agência sobre os indivíduos que os usam, e os aplicativos, enquanto artefatos, não estão fora desta lógica. À medida que se tornam familiares aos seus usuários, se intensifica o processo de alienação pelo qual os mesmos passam. Tais aplicativos são projetados de modo a prever comportamentos do usuário, antecipando seu estado emocional e promovendo estímulos sensoriais para manipulação fácil e rápida, características que dão ao produto a qualidade de uso intuitivo (Giacomin, 2014). Dessa maneira, sendo a análise semiótica uma interpretação consciente dos signos encontrados nas telas dos aplicativos iFood, demonstrou-se como as decisões e ações dos usuários são impactadas por conta das interpretações possíveis dentro daquele contexto.

Mesmo que as declarações das plataformas digam que elas são agentes neutros no processo de intermediação, a forma como seus aplicativos se estruturam e interagem com os usuários sugere outro cenário. Ao invés de intermediadores neutros, é possível considerá-los guias para seus usuários, pois indicam intencionalmente opções para que as tarefas almejadas sejam concluídas.

A partir das análises feitas, observou-se que há uma distinção muito clara na forma como a cada grupo de usuários é atribuído um papel específico. Enquanto o Cliente é sugerido como quem toma as decisões e tem muitas possibilidades de ação, o Entregador é visto como passivo, cumpridor de tarefas a quem são dadas poucas possibilidades de interferência no processo.

\section{Referências}

AIRBNB. Termos de serviços. 2020. Disponível em: https://www.airbnb.com.br/ terms. Acesso em: 20 jul. 2020.

BONSIEPE, G. Design, cultura e sociedade. São Paulo: Blucher, 2011. BONSIEPE, G. Design: do material ao digital. São Paulo: Blucher, 1997. CARDoso, R. Design para um mundo complexo. São Paulo: Ubu, 2016. Dopamine: uber [websérie]. Direção: Léo Favier. Produção: Loïc Bouchet. Paris: Arte Creative, 2019. Disponível em: https://www.arte.tv/fr/videos/o85801o07-A/dopamine/ Acesso em: 12 set. 2020.

FOKKINGA, S.; DESMET, P.; HEKKERT P. Impact-centered design: introducing an integrated framework of the psychological and behavioral effects of design. International Journal of Design, Taipei City, v. 14, n. 3, p. 97-116, 2020. Giacomin, J. What is human centred design? The Design Journal, [S.1.], v. 17, n. 4 , p. 606-623, 2014. DOI: 10.2752/175630614X14056185480186

GOOGLE. Economia digital: como os brasileiros têm procurado por produtos e serviços de startups durante a pandemia. 2020. Disponível em: https://www. thinkwithgoogle.com/intl/pt-br/tendencias-de-consumo/economia-digital- 
como-os-brasileiros-tem-procurado-por-produtos-e-servicos-de-startupsdurante-a-pandemia/. Acesso em: 22 jul. 2020.

GRABHER, G. et al. Uber-production: from global networks to digital platforms.

Environment and Planning A: Economy and Space, [S.l.], v. 52, n. 5, p. 10051016, 2020. DOI: 10.1177/0308518X20916507. Disponível em: https://journals. sagepub.com/doi/abs/10.1177/0308518X20916507?journalCode=epna\#articl eCitationDownloadContainer. Acesso em: 24 abr. 2020.

IBGE. Número de pessoas que trabalham em veículos cresce $29,2 \%$, maior alta da série. Agência IBGE Notícias. 2019. Disponível em: https://agenciadenoticias. ibge.gov.br/agencia-noticias/2012-agencia-de-noticias/noticias/264 24-numero-de-pessoas-que-trabalham-em-veiculos-cresce-29-maior-alta-daserie. Acesso em: 22 jul. 2020.

IFOOD. Termos e condições. 2020a. Disponível em: https://www.ifood.com.br/ termos. Acesso em: 20 jul. 2020.

IFOOD. Termos e condições de uso para entregadores. 2020b. Disponível em: https://entregador.ifood.com.br/termosdeuso/. Acesso em: 20 jul. 2020.

INSTITUTO FOODSERVICE BRASIL. Delivery fechamento 2019. 2019. Disponível em: https://9647e778-371d-421b-9615-c5e4af4ae440.filesusr.com/ugd/o5b3of_ ae37323b694449 a6bc5f62169e40d191.pdf?index=true. Acesso em: 22 jul. 2020.

ISLAM, M. N. A systematic literature review of semiotics perception in user interfaces. Journal of Systems and Information Technology, [S.l.], v. 15, n. 1, p. 45-77, 2013. DOI:10.1108/13287261311322585

KALBACH, J. Mapeamento de experiências: um guia para criar valor por meio de jornadas, blueprints e diagramas. Rio de Janeiro: Alta books, 2017.

KRUG, S. Don't make me think: a common sense approach to web usability. Berkeley, CA: New Riders, 2006.

MARK, R.; CARSTEN, S.; BASOLE, R. The digital platform: a research agenda. Journal of Information Technology, [S.1.], v. 33, p. 124-135, 2020. Disponível em: https://journals.sagepub.com/doi/pdf/10.1057/s41265-016-0033-3. Acesso em: 31 maio 2020.

MILLER, D. Trecos, troços e coisas: estudos antropológicos sobre a cultura material. Rio de Janeiro: Zahar, 2013.

MOVILE. iFood: como funciona a maior foodtech da América Latina. 2020. Disponível em: https://movile.blog/ifood-como-funciona-a-maior-foodtechda-america-latina/. Acesso em: 20 jul. 2020.

RAPPI. Termos e condições de uso da plataforma "Rappi”. 2020. Disponível em: https://legal.rappi.com/brazil/termos-e-condicoes-de-uso-da-plataformarappi/?_ga=2.12614 8237.1230472185.1595239352-36521163.1595239352\&_gac= 1.45361808.1595240418.EAIaIQobChMI95Whocnb6gIVEwSRCh2vUAej EAAYASAAEgK_gPD_BwE. Acesso em: 20 jul. 2020.

SANTAELla, L. Semiótica aplicada. São Paulo: Pioneira Thomson Learning, 2005. SILVEIRA, s. et al. Análise das plataformas de compartilhamento online e de suas práticas colaborativas. Revista EPTIC, São Cristóvão, v. 20, n. 2, 2018. Disponível em: https://seer.ufs.br/index.php/eptic/article/view/9610. Acesso em: 10 jun. 2020.

UBER. Quem somos. 2020. Disponível em: https://www.uber.com/br/pt-br/ about/. Acessado em: 20 jul. 2020. 
VAlEnTE, J. Plataformas digitais, economia e poder: apresentação do dossiê temático da revista EPTIC 2020-1. Revista EPTIC, v. 22, n. 1, 2020. Disponível em: https://seer.ufs.br/index.php/eptic/article/view/13032. Acesso em: 16 maio 2020.

\section{Sobre os autores}

\section{Ana Carolina Ribeiro Ferreira da Costa}

ana.ferreira.costa@alumni.usp.br

Mestranda do Programa de Pós-Graduação em Design,

Faculdade de Arquitetura e Urbanismo da Universidade de São Paulo R. do Lago, 876 - Butantã, São Paulo, SP, 05508-080

\section{Leandro Manuel Reis Velloso}

leandrovelloso@usp.br

Professor doutor do Departamento de Projeto,

Faculdade de Arquitetura e Urbanismo da Universidade de São Paulo R. do Lago, 876 - Butantã, São Paulo, SP, 05508-080

Artigo recebido em/Submission date: 1/10/2020

Artigo aprovado em/Approvement date: 3/5/2021 